\title{
Short time-lapse seismic repeatability test, CO2CRC Otway Project, Victoria, Australia
}

\author{
Yousuf Al-Jabri* and Milovan Urosevic, Exploration Geophysics Department, Curtin University of Technology \\ and CO2CRC.
}

\begin{abstract}
Summary
High seismic repeatability is critical to the monitoring program of the Naylor Field because of the small timelapse effect related to $\mathrm{CO}_{2}$ injection into depleted gas reservoir (Naylor). To be able to obtain reliable seismic from a time-lapse seismic survey, the repeatability of the time-lapse survey must be determined. This can be achieved by performing zero-time repeatability tests through the acquisition and reacquisition of data in the same area before any changes occur in the reservoir. By comparing the analysis of multiple repeated pre-stack 2D surface seismic and VSP data, differences maybe established to minimize near-surface system effects.
\end{abstract}

\section{Introduction}

The study of time-lapse seismic surveys is not equally cited using land verses marine data. More time-lapse seismic case histories are reported using offshore data such as fields with primary or water-flood production in North Sea and the Gulf of Mexico. A land time lapse seismic case histories are mainly from shallow reservoirs with thermal heavy oil production such as those in Canada and Indonesia. The major problem for time-lapse seismic is how to deal with the non-repeatability factors in both acquisition and processing data. For time-lapse seismic data acquisition, the main non-repeatability factors are related to the differences in geometries, sources, observation directions, geophone types, offsets of shot and receiver points, seismic crews and equipment as well as nearsurface conditions. For data processing, the nonrepeatability factors are associated with the differences in processing workflow, parameters, algorithms, precisions, and contractors (Ross, 1996 and Rickett, 1999). Over the past 10 years, several attributes have been proposed to quantify $4 \mathrm{D}$ repeatability at different stages in the $4 \mathrm{D}$ seismic workflow. Understanding these attributes and their inter relationships and impact on the final $4 \mathrm{D}$ products is critical to ensure business requirements are met. Careful consideration needs to be given to the balance between acquisition costs and likelihood of successfully detecting the reducing 4D signals as field life progresses.

Repeatability is the measure of consistency in acquisition and processed data. Ideal repeatability results from identical data acquisition, such that the difference between the two measurements is zero. However, seismic data processing is required to enhance repeatability when acquisition is less than ideal. This also raises the cost issue. A tedious data acquisition effort may be too costly and may not even be necessary. If we can estimate the magnitude of the changes we expect to see by carefully evaluating the geological and engineering data, we can design a seismic survey and a data processing flow to monitor the changes in the reservoir.

One way to test system repeatability is to perform a "zerotime repeatability test" by recording multiple datasets within a very short time interval before any changes occur in the reservoir. Another way to test repeatability is to examine differences between datasets on a horizon basis where conditions are known to be static. If the differences between the surveys are less than the expected differences in the reservoir, the system repeatability is favorable for interpretation. If the measured differences are larger than expected differences in the reservoir, then the data processing flow needs to be redesigned or the acquisition parameters need to be adjusted. Without zero-time repeatability tests, the accuracy of the observed differences can be questionable.

\section{Methodology}

We attempted to analyze the zero time-lapse of pre-stack seismic data of repeated shots along a 2D seismic test line. The data was recorded using a Mini-Vibroseis seismic source with a sweep $20-120 \mathrm{~Hz}$. The pilot trace was recorded at every shot position from an accelerometer fixed to its plate to correlate with the recorded data (uncorrelated data). In this study, we analyzed the NRMS difference and the amplitude spectra between the multiple repeated shots at the same location. This was carried out for the pilot traces, uncorrelated data, correlated data each shot with it recorded pilot, and all shots correlated with one selected pilot. We also attempted to analyse pre-stack VSP data recorded by the Mini-Vibroseis and pre-stack VSP data recorded using the Weight-drop. These VSP data were recorded 5 times at every single location and then stacked as one shot gather. However, we attempted to analyse the repeatability between the repeated 5 shots before stacking at every shot station. We also compared the applicability of two different sources for Naylor Field monitoring program. 


\section{Short time-lapse seismic repeatability test}

\section{Analysis and Results}

Studies were made of the seismic repeatability between the repeated shots 35,36 and 37 at the same shot position and between the repeated shots 38 and 39 at the same shot position and $10 \mathrm{~m}$ apart from the repeated shots during the acquisition of the 2D seismic test line in May 2006. We analysed the seismic repeatability of the pilot traces which recorded at every shot position using the accelerometer. We found that the NRMS difference between the repeated pilot traces at the same position is approximately $15-30 \%$ (Figure 1). We interpret these changes to be due to the change of the ground compression and radio transmission noise. By compression between these repeated pilots we found that pilot number 35 of shot gather 35 is more repeatable with other pilot traces of other shots and therefore pilot trace number 35 was selected to correlate all our data with this pilot to enhance the seismic repeatability. Correlating the vibroseis data with one selected pilot trace improved the seismic repeatability comparing with correlating the same data each shot with its own recorded pilot trace 5\% (Figure 2). Refraction data is the more repeatable seismic data than the reflection data and this was because the refraction data was the least affected by seismic noise. The VSP vibroseis data had a very high repeatability (approximately NRMS difference $\sim 1-4 \%$ ) while three repeated shots per shot station using vibroseis were sufficient to be stacked as one shot (Figure 3). The VSP weight-drop data had less repeatability (approximately NRMS difference 20\%) compared to the vibroseis data and by looking at the amplitude spectrum of the repeated shots of the weight-drop source, five repeated shots per shot station using the weight-drop was the minimum numbers of repeated shots to be stacked as one shot (Figure 4).

\section{Conclusion}

Time-lapse 3D technology at Naylor Field of the Otway Basin Pilot Project can produce results which can lead us to better reservoir management. However, one question that must be answered is how much residual reflection energy is tolerable to capture the expected changes of $\mathrm{CO} 2$ accumulation with a minimal cost. Zero-repeatability testing is important to evaluate the seismic monitoring. Correlating the Vibroseis data with one pilot enhanced the seismic repeatability. Near-surface compression has high impact in seismic repeatability. Min-Vibroseis is a more repeatable source than a weight-drop for the Naylor Field case. Five repeated shots per shot station using a weightdrop was the minimum number of repeated shots needed to be stacked whereas three repeated shots by a Mini-
Vibroseis source were more than sufficient. The NRMS difference is a function of the seismic processing window. VSP data provide a better seismic repeatability than surface seismic data.

\section{Acknowledgments}

This work is sponsored by CO2CRC and we thank S. Sharma and A. Kepic for making these experiments possible. We are grateful to our colleagues from Curtin University R. Pevzner, C. Dupuis and A. Dzunic for motivating discussions. Special thanks to Professor Brian Evens for providing useful comments and help in the editing process. 


\section{Short time-lapse seismic repeatability test}

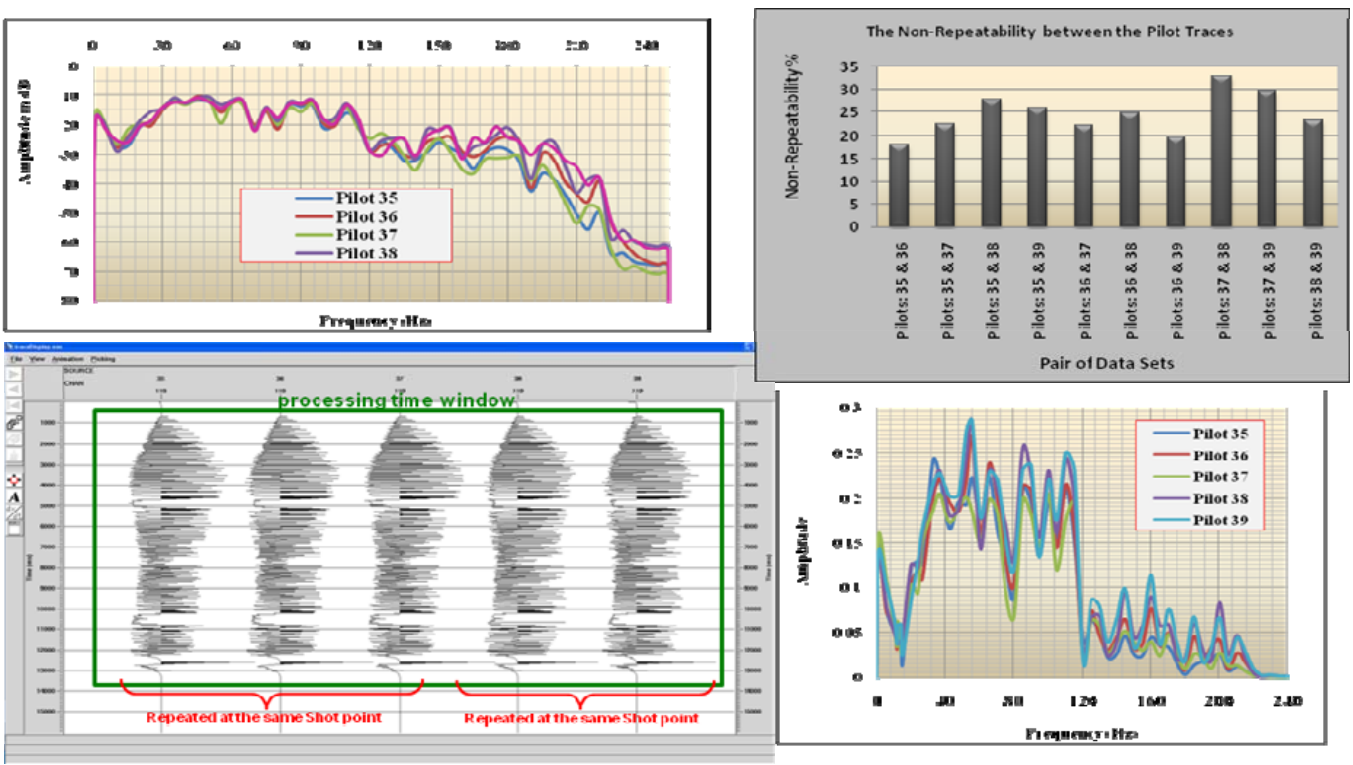

Figure 1: Amplitude spectra of the pilot traces of shot gathers 35, 36 and 37 which have been acquired at the same position 2D seismic line using a Mini-Vibroseis. Approximately 15-30\% of non-repeatability is due to the compression in the ground and noise due to the radio transmission. As well as shots 38 and 39 are acquired at the same position where $10 \mathrm{~m}$ apart from shots 35 , 36 and 37.
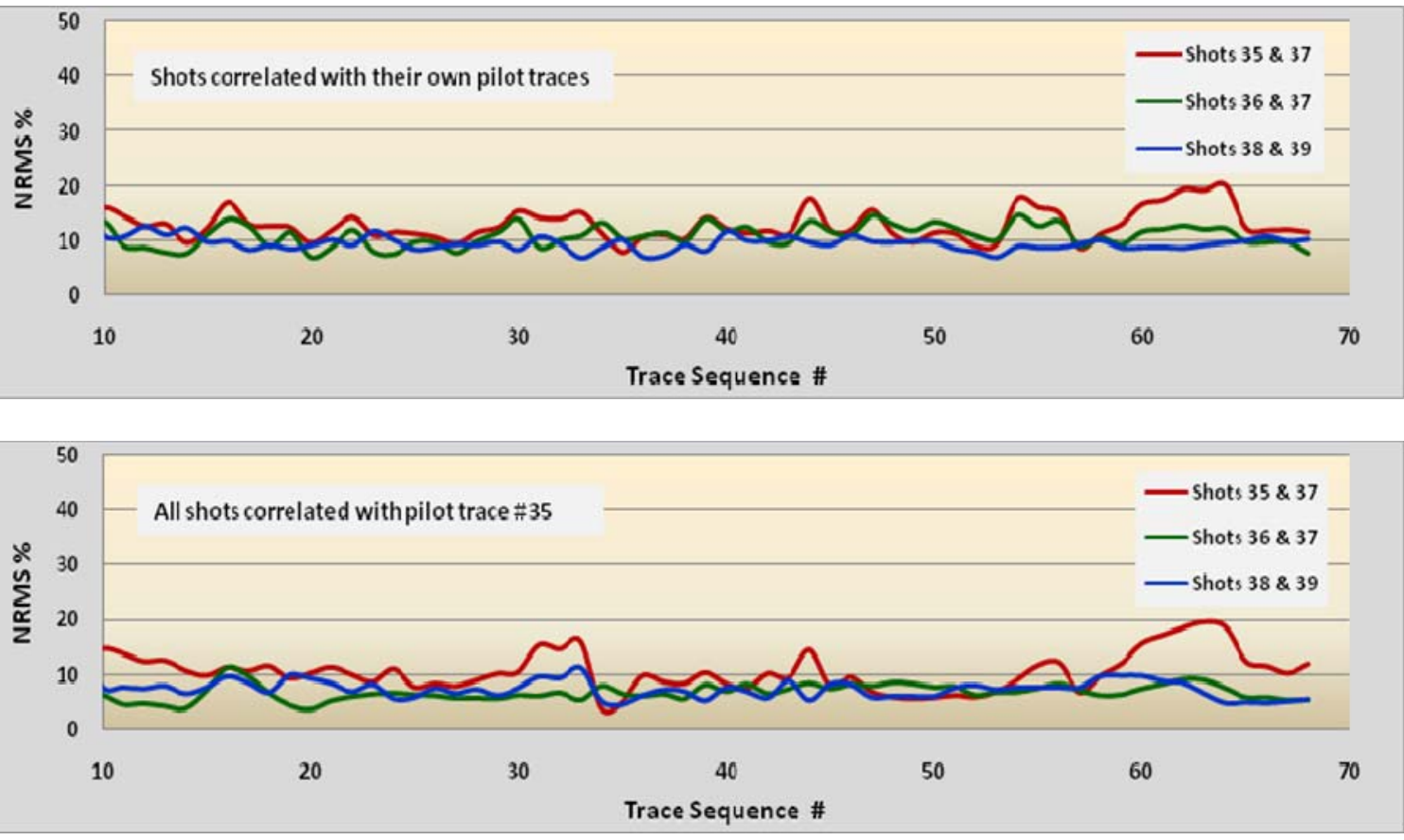

Figure 2: The NRMS difference between the correlated shot gathers 35-39 when they are correlated with their own pilot traces (top) and when they are correlated with one selected pilot trace number 35 (bottom) for window of 2000ms. The repeatability was enhanced when all shots were correlated with one pilot trace. 


\section{Short time-lapse seismic repeatability test}
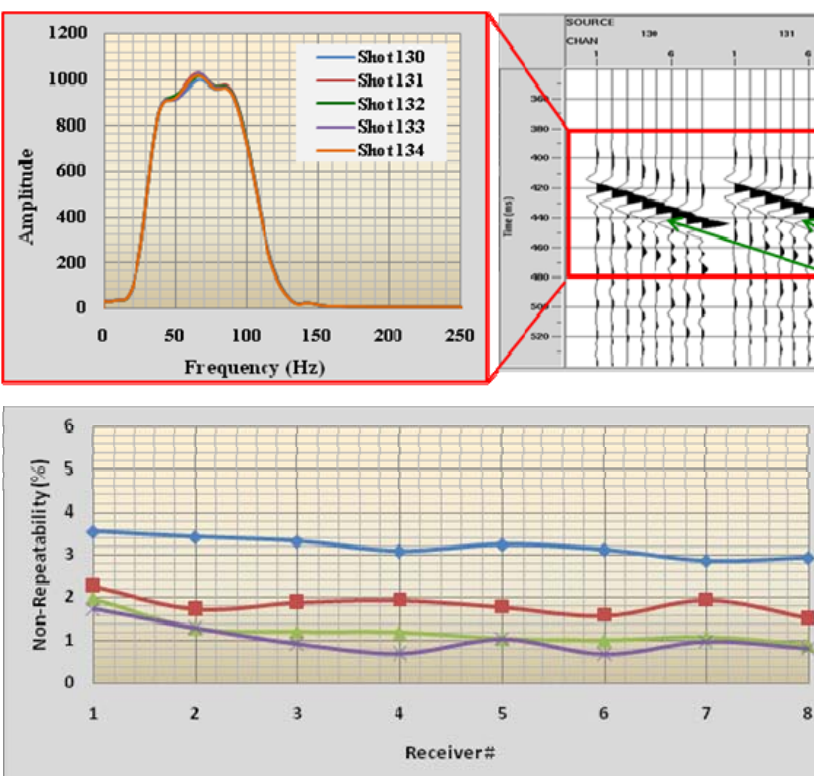

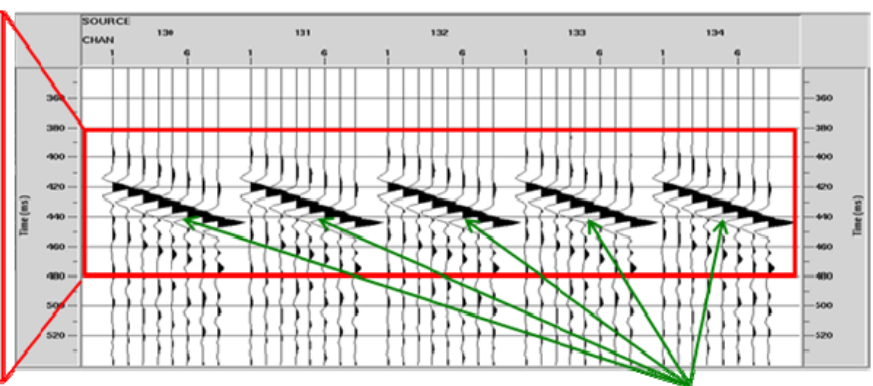

The RRMS maxued whith a wnilow of $50 \mathrm{~ms}$ Event above

Figure 3: The pre-stack statistical amplitude spectra (top left) of repeated five shots using Mini-Vibroseis at SP test of ZVSP (Zero-offset Vertical Seismic Profiling) with window of 100ms taken around the bright horizon. All shots have the same amplitude spectra. All shots were compared with the last shot when the near surface became more compacted. The NRMS difference between theses shots is decreasing as the number of shots increased.

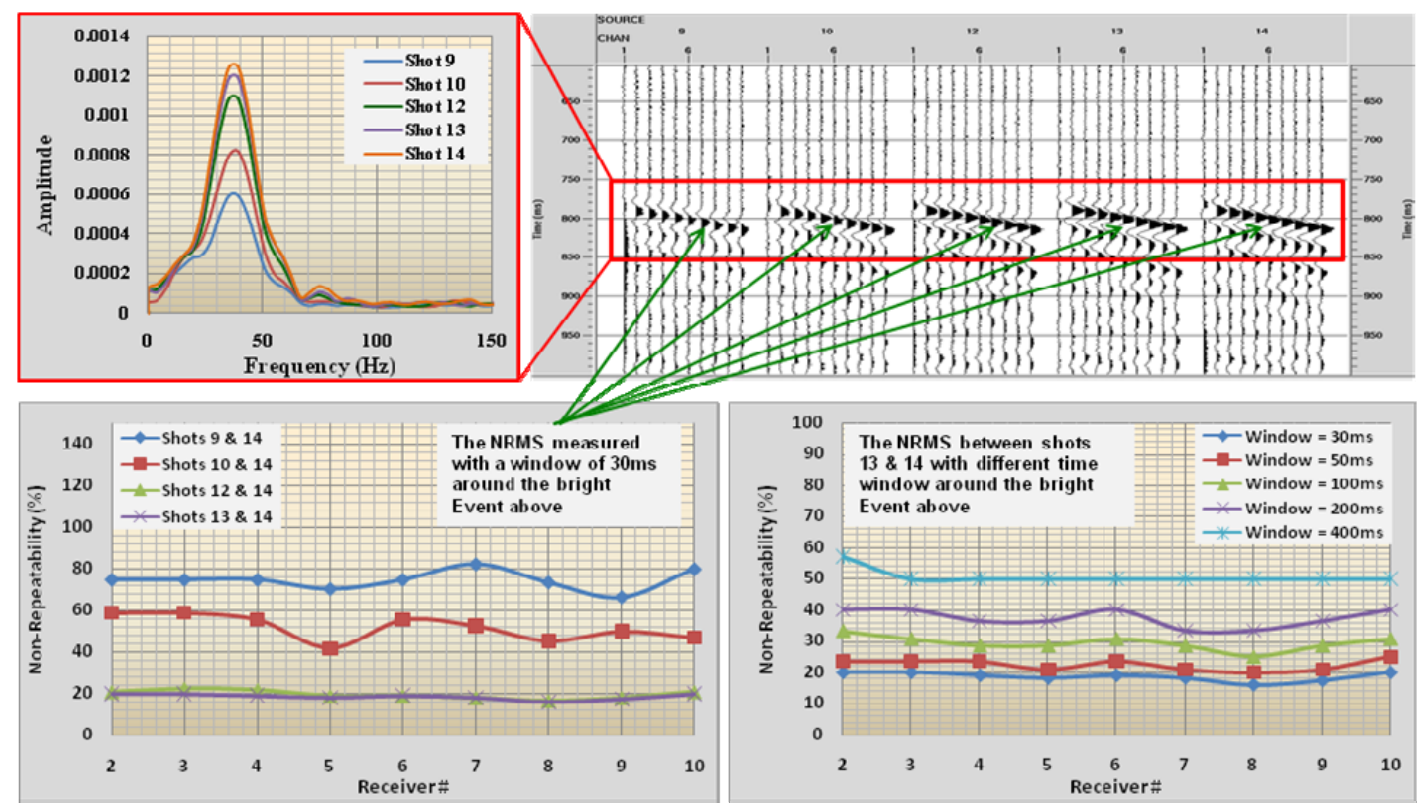

Figure 4: The pre-stack statistical amplitude spectra (top left) of repeated five shots using Weight-Drop at SP1075 of 3DVSP with window of $100 \mathrm{~ms}$ taken around the bright horizon. The amplitude spectra are increasing as the number of shots increased due to the ground compaction. All shots were compared with the last shot when the near surface became more compacted; the NRMS difference between theses shots is decreases as the number of shots increased. 


\section{EDITED REFERENCES}

Note: This reference list is a copy-edited version of the reference list submitted by the author. Reference lists for the 2010 SEG Technical Program Expanded Abstracts have been copy edited so that references provided with the online metadata for each paper will achieve a high degree of linking to cited sources that appear on the Web.

\section{REFERENCES}

Calvert, R. W., P. Hatchell, and C. Jones, 2002, 4D acquisition geometry requirements and QA: 64th EAGE Conference, Extended Abstracts, A017.

Campbell, S., T. A. Ricketts, D. M. Davies, C. P. Slater, G. G. Lilley, J. Brain, J. Stammeijer, and A. C. Evans, 2005, Improved 4D seismic repeatability - a west of Shetlands towed streamer acquisition case history: 75th Annual International Meeting, SEG, Expanded Abstracts, 2394-2397.

Rickett, J. E., and D. E. Lumley, 2001, Cross-equalization data processing for time-lapse seismic reservoir monitoring: A case study from the Gulf of Mexico: Geophysics, 66, 1015-1025, doi:10.1190/1.1487049.

Ross, C. P., G. B. Cunningham, and D. P. Weber, 1996, Inside the cross-equalization black box: The Leading Edge, 15, 1233-1240, doi:10.1190/1.1437231. 